\title{
Laterality effects in symbolic judgment: The influence of semantic congruity on hemispheric processing
}

\author{
HEDY WHITE \\ Western Carolina University, Cullowhee, North Carolina \\ WILLIAM P. BANKS \\ Pomona College and Claremont Graduate School, Claremont, California \\ and \\ ERAN ZAIDEL \\ University of California, Los Angeles, California
}

\begin{abstract}
In two experiments, we used lateralized versions of the comparative judgment paradigm to investigate the effects of semantic congruity on hemispheric processing of symbolic information. When the comparative adjectives used in instructions were congruent with the scale end from which stimulus terms were drawn, reaction times were shorter when the hemisphere to which the terms were lateralized also controlled the hand used to register the response than when instructions and stimuli were semantically incongruent. These laterality-effect patterns suggest that hemispheric cooperation increases processing efficiency.
\end{abstract}

In the research reported in this article, we investigated the role of the human cerebral hemispheres in making symbolic judgments of serial order. These judgments require subjects to determine the relative position of items in an ordered series, where items differ from each other in some quantifiable dimension. We focused on a robust finding in the comparative judgment literature (e.g., Banks \& White, 1985; Holyoak \& Mah, 1981), the semantic congruity effect, which is an interaction of the comparative adjective used in the instructions in comparative judgment tasks with the scale end from which the stimuli to be compared are drawn. When the comparative adjective and the stimuli are both from the marked end or the unmarked end of the continuum (i.e., congruent combinations), reaction times (RTs) are generally shorter than when the marking of instructions and stimuli differ (incongruent combinations). For example, the congruity effect would be found if discriminating between the terms ANT and FLEA on the dimension of size is faster when instructions are to choose the smaller term than when instructions are to choose the larger term.

Caramazza, Gordon, Zurif, and DeLura (1976) found that right-hemisphere ( $\mathrm{RH})$-damaged patients and control subjects with no damage (left-damaged patients were not used, but see their footnote 5, p. 45) were equally accurate (latency was not measured) in processing congruent combinations but the patients were less accurate than the

Requests for reprints should be addressed to Hedy White, Department of Psychology, Western Carolina University, Cullowhee, NC 28723. control subjects in processing incongruent combinations. These findings imply that the left hemisphere (LH) has access to sufficient semantic information to enable independent processing of congruent combinations, but for incongruent combinations, processing is more efficient when the resources of the two hemispheres are shared.

To extend these findings, in the two experiments reported below, we used a lateralized version of the timed symbolic judgment paradigm. For congruent combinations, the results of Caramazza et al. (1976) suggest that either hemisphere should be able to accomplish the entire task independently. Such a finding would be supported by an interaction between visual field of stimulus presentation and the hand used to respond (e.g., see Moscovitch, Scullion, \& Christie, 1976; Zaidel, 1985). That is, RTs should be shorter when the stimuli are presented to the same hemisphere that issues the response command, since when the contralateral hand must respond, transfer to the opposite hemisphere for response programming should lengthen the RT. Thus, when stimuli are directed to the LH (presented in the right visual hemifield, RVF), RTs should be shorter when the right hand responds than when the left hand responds, and, similarly, RH (left visual hemifield, LVF) trials should be shorter when the left hand responds than when the right hand responds. The two present experiments showed this interaction for congruent combinations.

An opposite form of interaction, in which contralateral combinations (LVF-right hand and RVF-left hand) are processed faster than ipsilateral ones (LVF-left hand and RVF-right hand), has been interpreted as suggesting that processing efficiency increases when the resources of the 
hemispheres are shared (e.g., see Alwitt, 1981; Green, 1984; Wickens \& Sandry, 1982). The reasoning is that when a single hemisphere must accomplish an entire task, processing demands may create intrahemispheric interference, which reduces efficiency. Thus, RTs may be shorter when one hemisphere is responsible for stimulus processing and the other is responsible for response programming. According to Green (1984), ipsilateral advantages would be expected when processing demands are low, and contralateral advantages would be expected when processing demands are increased. In the present research, contralateral combinations of incongruent stimuli tended to be processed faster than ipsilateral ones, although the difference was reliable only in Experiment 2.

\section{EXPERIMENT 1}

\section{Method}

Subjects. The subjects in this approximately 40 -min experiment were 20 male and 20 female right-handed undergraduates from introductory psychology courses.
Procedure. The subjects were tested on timed comparative judgments of the relative magnitude of digits. Instructions and digit pairs were presented to the subjects on an Amdek black-and-white monitor controlled by an Apple II + microcomputer. On each trial, instructions ("Bigger?" or "Smaller?") appeared in the center of the screen for $500 \mathrm{msec}$ and were followed by a 500 -msec central fixation dot. Immediately after the fixation dot, two digits (presented horizontally) appeared for $50 \mathrm{msec}$ either in the center of the screen or to the left or right of fixation. The subject responded by pressing a key on the side of the response box (placed at midline) corresponding to the position of the digit specified by the instructions. The subject's response caused the computer to present feedback ("correct" or "incorrect") and begin a new trial. The computer recorded correct RTs and number of errors (but not RTs for errors), and repeated trials that resulted in errors later in a block.

The subjects received four experimental blocks of 72 RT trials each, two in which the right hand operated the response box and two in which the left hand operated it. Order of using the right and left hand was counterbalanced over subjects (separately for each sex), with half of the subjects using a right, left, left, right order and half using a left, right, right, left order. Order of receiving trials in blocks was randomized by the computer individually for each subject. The stimuli were 12 pairs of digits: $1-2,1-3,1-4,2-3,2-4,2-5,8-9,7-9,6-9,7-8,6-8$, and 5-8. They appeared in the following orthogonal conditions: 2 response hands (right vs. left) $\times 3$ visual fields (right, left, and center) $\times 2$

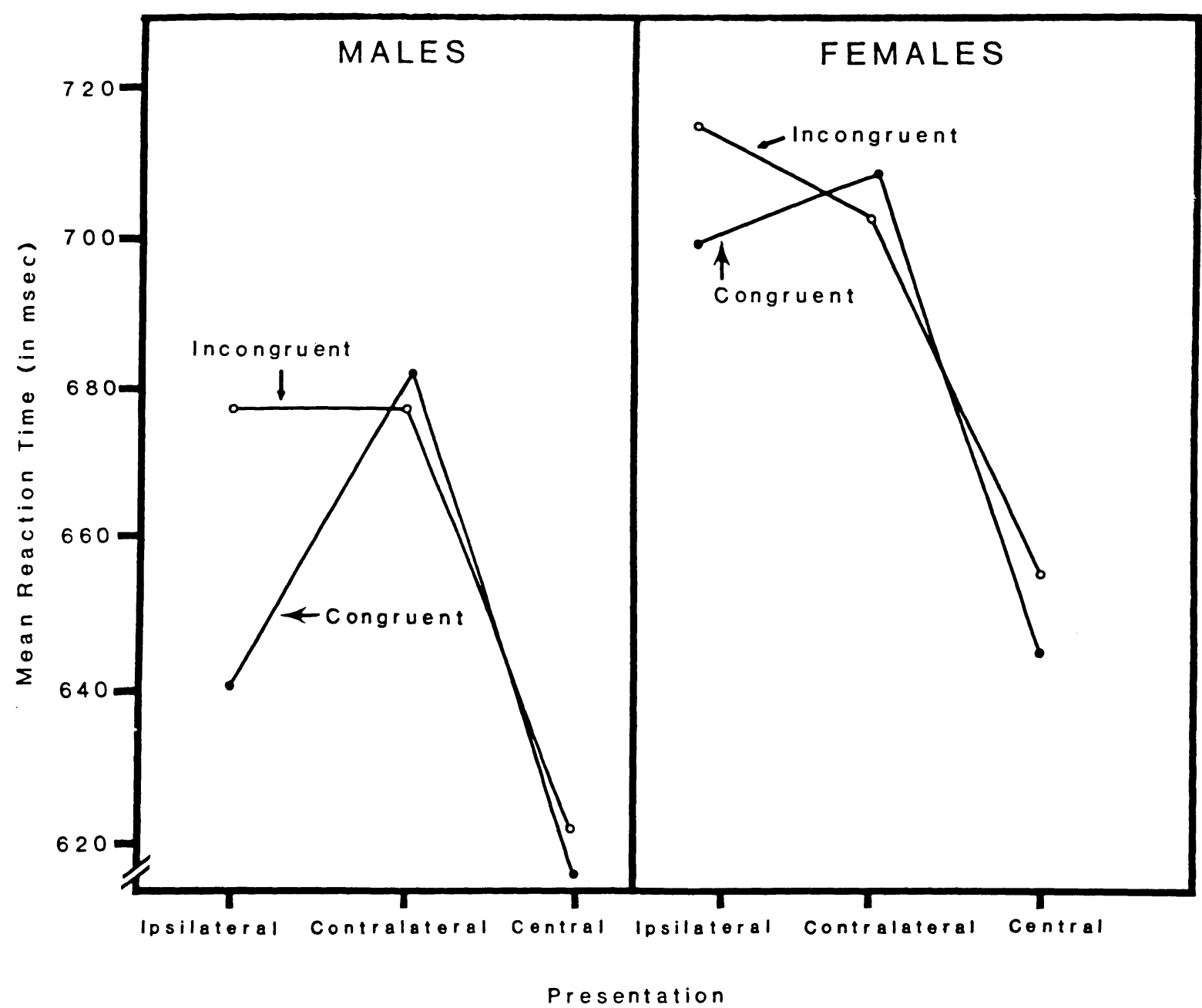

Figure 1. Mean reaction times for magnitude judgments of digits in Experiment 1 for males (left panel) and females (right panel) plotted for ipsilateral, contralateral, and central presentations of congruent and incongruent instruction-stimulus combinations. 
stimulus-instruction combinations (congruent combinations are pairs containing the digit 1 or 2 combined with "choose smaller" instructions or pairs containing the digit 8 or 9 with "choose larger" instructions; incongruent ones are pairs with the small digits with "choose larger" or large pairs with "choose smaller") $\times 2$ permutations of each pair. The two blocks in each hand condition used a different permutation of a pair, with order of receiving these blocks counterbalanced over subjects (separately for each sex $\times$ hand-order condition).

Prior to receiving the first and second experimental blocks, the subjects received a practice block of 36 RT trials (12 in each field), which used digit combinations not used in the experimental trials. On each practice block, the subjects used the hand they would use on the experimental block that followed.

\section{Results}

Figure 1 shows that for males (left panel), congruent pairs were processed $39 \mathrm{msec}$ more quickly in ipsilateral than in contralateral conditions $[F(1,28)=16.88$, $p<.01$ ]. Mean RTs for congruent pairs were $39 \mathrm{msec}$ shorter in the RVF-right-hand condition than in the RVF-left-hand condition, and they were also $39 \mathrm{msec}$ shorter in the LVF-left-hand condition than in the LVF-right-hand condition. For incongruent combinations, there was neither an ipsilateral nor a contralateral advantage. For females (Figure 1, right panel), on congruent combinations, ipsilateral presentations were processed only $7 \mathrm{msec}$ faster than contralateral presentations $(F<1.00)$, and the ipsilateral advantage occurred only for RVF presentations. For incongruent combinations, contralateral trials were processed faster (by $22 \mathrm{msec}$ ) than ipsilateral ones, but this effect was not statistically significant $[F(1,28)=1.61, p>.10]$.

In the overall ANOVA, the sex $\times$ congruity $\times$ hand $\times$ field interaction seen in Figure 1 was not reliable $(F<1.00)$. But the congruity $\times$ field $\times$ hand interaction was reliable $[F(1,38)=9.06, p<.01]$. There were

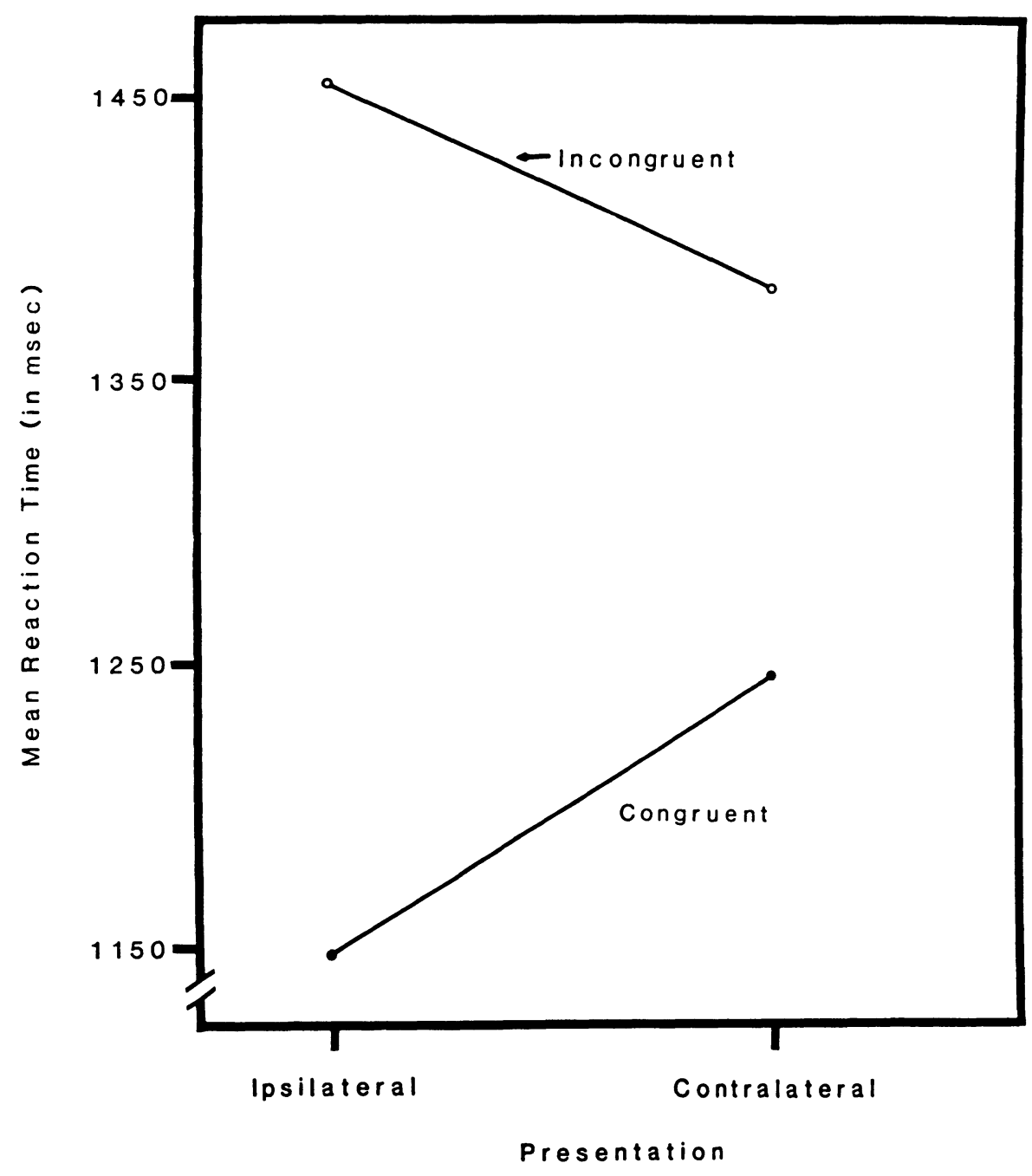

Figure 2. Mean reaction times (RTs) for comparative judgments plotted for each laterality condition in Experiment 2. The plot shows RTs for congruent and incongruent combinations for pairs with an end term (i.e., pairs in which one term refers to the smallest or largest animal in a 9-term series). 
no other reliable effects or interactions involving the laterality variables. Mean errors for the 40 subjects were $2.8 \%$, and errors and RTs were positively correlated (over the main variables in the design, $r=.66$ ).

\section{EXPERIMENT 2}

\section{Method}

Subjects. The subjects were 24 right-handed males from the same pool that was used in Experiment 1.

Procedure. The subjects were tested on timed comparative judgments of terms drawn from a 9-term series of names of animals ordered in size. Size ratings for these terms (based on a 1-20 scale) were obtained from 32 subjects who did not participate in either experiment. The terms (with mean ratings in parentheses) were: ANT (1.60), MOUSE (4.53), RAT (5.43), DUCK (6.80), SHEEP (9.73), DEER (11.07), COW (12.80), HIPPO (16.30), and WHALE (18.97). Referring to terms by their ordinal position in the 9-term ordering (e.g., $1=$ ANT , . ., $9=$ WHALE), there were 12 pairs, which corresponded with those used in Experiment 1. Instructions and stimulus pairs were presented as in Experiment 1, except that one term appeared on top of the other, distance from the fixation dot to the terms was measured from the first letter of the terms in each field, and the central condition was not used. Also, in this experiment, the stimulus terms remained in view for $120 \mathrm{msec}$. The subjects received two experimental blocks of $48 \mathrm{RT}$ trials each, one in which the right hand was used to respond and one in which the left hand was used. Different permutations of the pairs were used in each block, resulting in four counterbalanced conditions ( 2 hand orders $\times 2$ permutations) with 6 subjects in each.

Before receiving the first experimental block, each subject received two practice blocks of $\mathbf{4 8}$ trials each, one in which the left hand was used to respond and one in which the right hand was used. Practice and experimental blocks used the same stimuli. Prior to receiving practice trials, the subjects were shown the 9-term ordering. A few subjects were unsure of the relative positions of DEER and COW but they agreed to assume the given order for the purpose of the experiment.

\section{Results}

In this experiment, the ipsilateral advantage for congruent combinations was stronger for pairs with end terms than for pairs without end terms. Figure 2 plots the interaction using only pairs with end terms. For congruent combinations using these pairs, there was a $97-\mathrm{msec}$ ipsilateral advantage $[F(1,22)=11.32, p<.01]$ : RTs were 107 msec shorter under RVF-right-hand presentations than under RVF-left-hand presentations, and they were $88 \mathrm{msec}$ shorter under LVF-left-hand presentations than under LVF-right-hand presentations. For incongruent combinations, there was a $69-\mathrm{msec}$ contralateral advantage $[F(1,22)=5.73, p<.05]$ : RTs were $19 \mathrm{msec}$ shorter under RVF-left-hand presentations than under RVF-right-hand presentations, and they were $119 \mathrm{msec}$ shorter under LVF-right-hand presentations than under
LVF-left-hand presentations. For pairs without end terms, the ipsilateral advantage for congruent combinations was reduced to $10 \mathrm{msec}$, and for incongruent combinations, RTs were virtually identical under the two presentations (a 1 -msec ipsilateral advantage; $F \mathrm{~s}<1.00$ ). In the overall analysis, the interaction of laterality, congruity, and endedness was reliable $[F(1,22)=9.56, p<.01]$. Finally, the mean error rate for the 24 subjects was $8.7 \%$. There was a positive correlation of errors with RTs (over the main variables in the design, $r=.72$ ).

\section{DISCUSSION}

The two present experiments suggest that each hemisphere is able to process symbolic information independently when instructions and stimulus pairs are semantically congruent. Semantically incongruent combinations of stimuli and instructions, on the other hand, may be processed more efficiently when a single hemisphere is not responsible for both stimulus processing and response programming. Intrahemispheric interference on ipsilateral presentations may be attributable to the need for a congruity-ensuring transformation (see Banks, 1977), which would increase the hemispheric resources needed for processing.

\section{REFERENCES}

ALwIrT, L. F. (1981). Two neural mechanisms related to modes of selective attention. Journal of Experimental Psychology: Human Perception \& Performance, 7, 324-332.

BANKs, W. P. (1977). Encoding and processing of symbolic information in comparative judgments. In G. H. Bower (Ed.), The psychology of learning and motivation (Vol. 11, pp. 101-159). New York: Academic Press.

Banks, W. P., White, H. (1985). Semantic congruity and expectancy as separate processes. Memory \& Cognition, 13, 485-493.

Caramazza, A., Gordon, J., Zurif, E. B., \& Delura, D. (1976). Right-hemispheric damage and verbal problem solving behavior. Brain \& Language, 3, 41-46.

GrEEN, J. (1984). Effects of intrahemispheric interference on reaction time to lateral stimuli. Journal of Experimental Psychology: Human Perception \& Performance, 10, 292-306.

HolyoAK, K. J., \& MAH, W. A. (1981). Semantic congruity in symbolic comparisons: Evidence against an expectancy hypothesis. Memory \& Cognition, 9, 197-204.

Moscovitch, M., Scullion, D., \& Christie, D. (1976). Early versus late stages of processing and their relation to functional hemispheric asymmetries in fact recognition. Journal of Experimental Psychology: Human Perception \& Performance, 2, 401-416.

WICKENS, C. D., \& SANDRY, D. L. (1982). Task-hemispheric integrity in dual task performance. Acta Psychologia, 52, 227-247.

ZAIDEL, E. (1985). Callosal dynamics and right hemisphere language. In F. Lepore, M. Ptito, \& H. H. Jasper (Eds.), Two hemispheresone brain. New York: Alan R. Liss.

(Manuscript received February 20, 1990.) 